\title{
Overview of 5G Security Challenges and Solutions
}

\author{
Ijaz Ahmad, Tanesh Kumar, Madhusanka Liyanage, Jude Okwuibe, Mika Ylianttila, Andrei Gurtov
}

\begin{abstract}
The next generation networks (5G) will use novel technological concepts to meet the requirements of broadband access everywhere, high user and device mobility, and connectivity of massive number of devices (e.g. Internet of Things (IoT)) in an ultra-reliable and affordable way. Software Defined Networking (SDN) and Network Function Virtualization (NFV) leveraging the advances in cloud computing such as Mobile Edge Computing are the most sought out technologies to meet these requirements. However, securely using these technologies and providing user privacy in future wireless networks are the new concerns. Therefore, this paper provides an overview of the security challenges in clouds, SDN and NFV, and the challenges of user privacy. Henceforth, this paper presents solutions to these challenges and future directions for secure $5 \mathrm{G}$ systems.
\end{abstract}

Index Terms-Security; 5G Security; SDN; NFV; Cloud; Privacy; Standardization

\section{INTRODUCTION}

According to 5G-PPP (5G-Public Private Partnership), 5G will connect about 7 trillion wireless devices or things, shrink the average service creation time from 90 hours to 90 minutes and enable advanced user controlled privacy [1]. By connecting all aspects of life, $5 \mathrm{G}$ aims a digital society that requires high service availability and security using a diverse set of technologies. Therefore, the concepts of cloud computing, Software Defined Networking (SDN), and Network Function Virtualization (NFV) are sought out to meet the growing user and service demands within the constraints of Capital Expenditures (CapEx) and Operational Expenses (OpEx) through flexible network operation and management.

Cloud computing provides an efficient way for operators to maintain data, services and applications by bringing tech- nologically distinct systems into a single domain on which multiple services can be deployed to achieve a higher degree of flexibility and availability with less CapEx and OpEx. Multiaccess Edge Computing (MEC), using the concepts of cloud computing, will empower the network edge to process delay sensitive and context-aware applications in close proximity of users or things. Softwarizing network functions will enable portability and flexibility of networking systems and services. SDN enables network function softwarization by separating the network control from the data forwarding planes, and enabling programmability of both planes. Hence, SDN brings innovation in networking through abstraction and programmability on one hand and simplifies network management through logically centralized control of the network on the other hand.

NFV provides the basis for placing various network functions in different network perimeters and eliminates the need for function or service-specific hardware. SDN and NFV, complementing each other, improve network elasticity, simplify network control and management, break the barriers of vendorspecific proprietary solutions, and thus are considered the core technologies in the transformation of networks for $5 \mathrm{G}$ by $5 \mathrm{G}$ PPP. Network slicing, leveraging NFV and SDN, enables the $5 \mathrm{G}$ network infrastructure to share the same resources for mutliple use cases such as Internet of Things (IoT), enhanced broadband, and critical communication [1]. A generic 5G deployment scenario using these key technologies is depicted in Fig. 1.

However, recent research in these technologies reveal potential security challenges that must be addressed in order to ensure security of new 5G services and infrastructures,

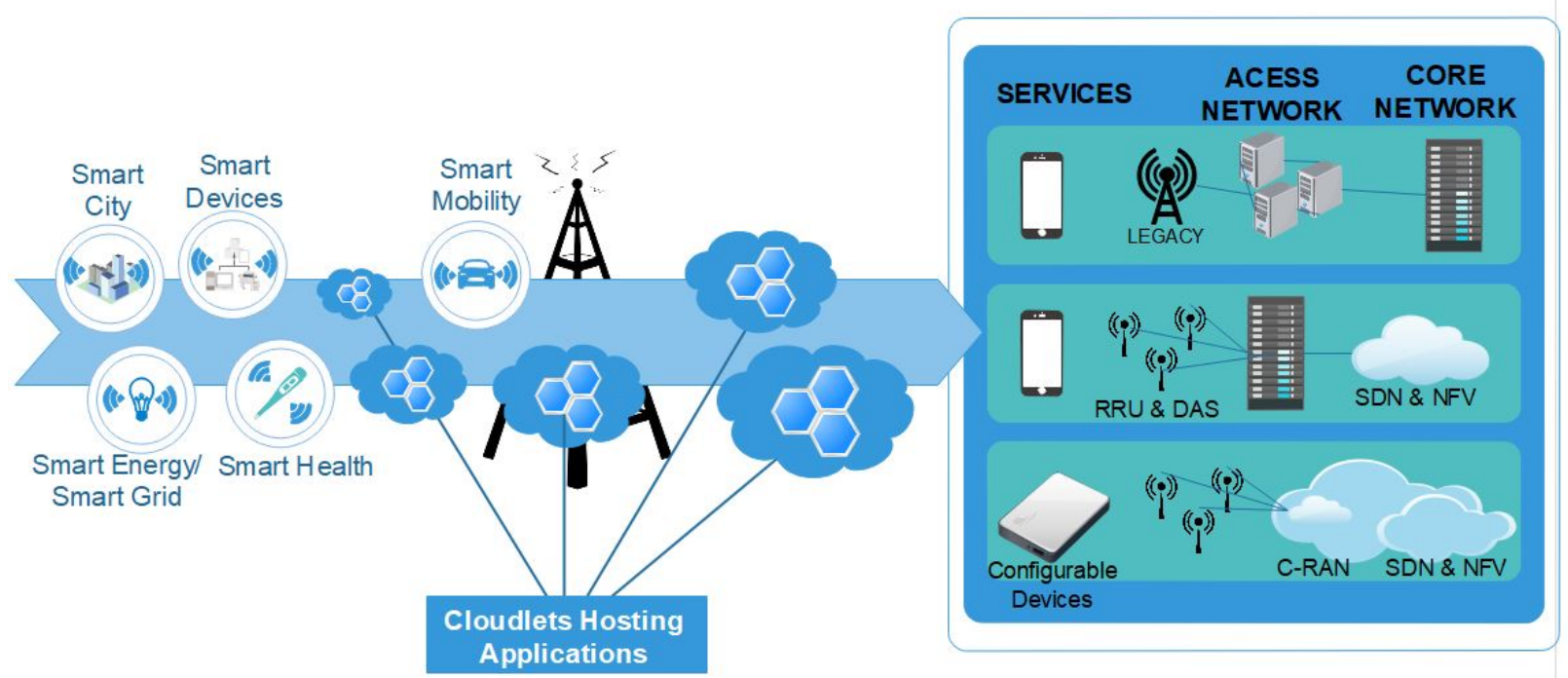

Fig. 1: 5G deployment scenarios and key technologies. 
and users. For example, multi-tenant shared cloud infrastructures among multiple virtual network operators require strict isolation at multiple levels to avoid illegal resource consumption and maintain integrity of users information of different operators [2]. According to the 5G PPP Phase 1 Security Landscape [1], network slicing has several open security challenges such as security isolation of network slices and security of inter-slice communications [3]. Moreover, programmable network architectures like SDN require strong authentication and authorization for applications to avoid misuse of the network resources exposed to applications through the control plane. Similarly, mis-configurations of VNFs (Virtual Network Functions) can lead to inter-federated conflicts creating geopardy in the whole network [4]. Since 5G will connect every aspect of life to the network having most of users' information stored and shared online, maintaining user privacy will be highly challenging.

Furthermore, wireless communication systems have been prone to security vulnerabilities from the very inception. In the first generation (1G) wireless networks, mobile phones and wireless channels were targeted for illegal cloning and masquerading. In the second generation (2G) of wireless networks, message spamming became common not only for pervasive attacks but injecting false information or broadcasting unwanted marketing information. In the third generation (3G) wireless networks, IP-based communication enabled the migration of Internet security vulnerabilities and challenges in the wireless domains. With the increased necessity of IP based communication, the fourth Generation $(4 \mathrm{G})$ mobile networks enabled the proliferation of smart devices, multimedia traffic, and new services into the mobile domain [5]. This development led to more complicated and dynamic threat landscape. With the advent of the fifth generation $(5 \mathrm{G})$ wireless networks, the security threat vectors will be bigger than even before with greater concern for privacy.

Therefore, it is crucial to highlight the security challenges that are threatening not only due to the wireless nature of mobile networks, but exist in the potential technologies that are highly important for 5G. The rest of the paper is organized as follows: Section II describes the key security challenges followed by security solutions for the highlighted security challenges in Section III. Section IV highlights the 5G security standardization activities at the time of writing this paper, and finally we conclude in Section V.

\section{Key Security Challenges IN 5G}

$5 \mathrm{G}$ needs robust security architectures and solutions since it will connect every aspect of life to communication networks. Therefore, we investigate and highlight the important security and privacy challenges in 5G networks (depicted in Fig. 2) and overview the potential solutions that could lead to secure $5 \mathrm{G}$ systems. The basic challenges in 5G highlighted by Next Generation Mobile Networks (NGMN) [6] and highly discussed in the literature are as follows:

- Flash network traffic: High number of end-user devices and new things (IoT).

- Security of radio interfaces: Radio interface encryption keys sent over insecure channels.

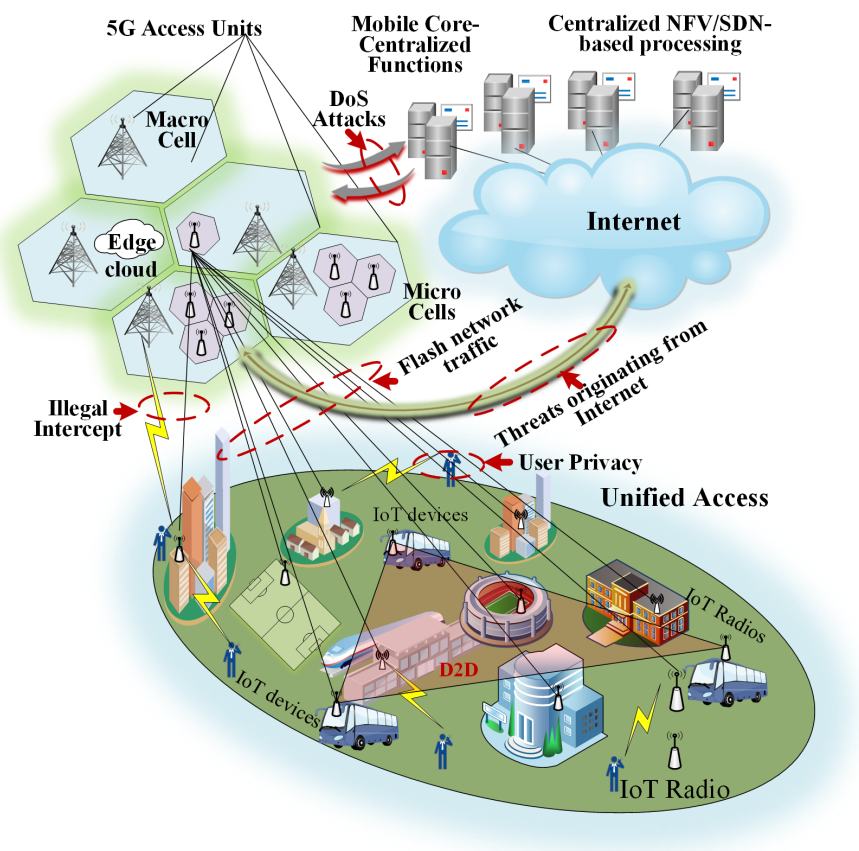

Fig. 2: 5G network and the threat landscape.

- User plane integrity: No cryptographic integrity protection for the user data plane.

- Mandated security in the network: Service-driven constraints on the security architecture leading to the optional use of security measures.

- Roaming security: User-security parameters are not updated with roaming from one operator network to another, leading to security compromises with roaming.

- Denial of Service (DoS) attacks on the infrastructure: Visible nature of network control elements, and unencrypted control channels.

- Signaling storms: Distributed control systems requiring coordination, e.g. Non-Access Stratum (NAS) layer of Third Generation Partnership Project (3GPP) protocols.

- DoS attacks on end-user devices: No security measures for operating systems, applications, and configuration data on user devices.

The 5G design principles outlined by NGMN beyond radio efficiency are: creating a common composable core and simplified operations and management by embracing new computing and networking technologies. Therefore, we focused on the security of those technologies that will fulfill the design principles outlined by NGMN i.e. mobile clouds, SDN and NFV. Table 1 provides a summary of different types of security threats and attacks, the targeted elements or services in a network, and the technologies that are most prone to the attacks or threats are tick-marked. These security challenges are briefly described in the following sections.

\section{A. Security Challenges in SDN}

SDN facilitates innovation in communication networks and simplifies network management by enabling programmability 
TABLE I: Security challenges in 5G technologies [7].

\begin{tabular}{|l|l|c|c|c|c|c|}
\hline \multirow{2}{*}{ Security Threat } & \multirow{2}{*}{ Target Point/Network Element } & \multicolumn{2}{|c|}{ Effected Technology } & \multirow{2}{*}{ Links } & \multirow{2}{*}{ Privacy } \\
\hline & & SDN & NFV & Cloud & & \\
\hline DoS attack & Centralized control elements & $\checkmark$ & $\checkmark$ & $\checkmark$ & & \\
\hline Hijacking attacks & SDN controller, hypervisor & $\checkmark$ & $\checkmark$ & & & \\
\hline Signaling storms & 5G core network elements & & & $\checkmark$ & $\checkmark$ & \\
\hline Resource (slice) theft & Hypervisor, shared cloud resources & & $\checkmark$ & $\checkmark$ & & \\
\hline Configuration attacks & SDN (virtual) switches, routers & $\checkmark$ & $\checkmark$ & & & \\
\hline Saturation attacks & SDN controller and switches & $\checkmark$ & & & & \\
\hline Penetration attacks & Virtual resources, clouds & $\checkmark$ & & $\checkmark$ & & \\
\hline User identity theft & User information data bases & & & $\checkmark$ & & $\checkmark$ \\
\hline TCP level attacks & SDN controller-switch communication & $\checkmark$ & & & $\checkmark$ & \\
\hline Man-in-the-middle attack & SDN controller-communication & $\checkmark$ & & & $\checkmark$ & \\
\hline Reset and IP spoofing & Control channels & & & & $\checkmark$ & \\
\hline Scanning attacks & Open air interfaces & & & & $\checkmark$ & $\checkmark$ \\
\hline Security keys exposure & Unencrypted channels & & & & $\checkmark$ & \\
\hline Semantic information attacks & Subscriber location & & & & $\checkmark$ & $\checkmark$ \\
\hline Timing attacks & Subscriber location & & & $\checkmark$ & & $\checkmark$ \\
\hline Boundary attacks & Subscriber location & & & & & $\checkmark$ \\
\hline IMSI catching attacks & Subscriber identity & & & & $\checkmark$ & $\checkmark$ \\
\hline
\end{tabular}

and logically centralizing the network control planes. These two features are significantly important for future networks, however, they also open the network to security challenges. For example, the SDN controller updates or modifies flow rules in the data forwarding elements. This control information traffic can be easily identified making it a visible entity in the network and rendering it a favorite choice for DoS attacks. Similarly, the centralization of network control can also make the controller a bottleneck for the whole network in case of saturation attacks. By enabling programmability, most network functions can be implemented as SDN applications. If malicious applications are granted access or critical Application Programming Interfaces (APIs) are exposed to unintended software, a havoc can be spread across the network [7].

The current SDN architecture i.e. OpenFlow, requires the data forwarding elements to store traffic flow requests until the controller updates the flow forwarding rules. Hence, the data plane elements can also be prone to saturation attacks since the forwarding elements, such as OpenFlow switches, have limited resources to buffer unsolicited (TCP/UDP) flows. Furthermore, this dependency on the controller requires the control-data planes channel to be resilient to security attacks unlike the current optional use of security protocols and long restoration delays in large networks. Redundant or multiple controllers may solve the challenge of controller availability or increase resilience to security attacks, however, misconfiguration of forwarding elements or inter-federated conflicts due to multiple controllers will hinder network-wide security policy enforcement [4].

\section{B. Security Challenges in NFV}

Even though NFV is highly important for future communication networks, it has basic security challenges such as confidentiality, integrity, authenticity and non-repudiation [7]. From the point of view of its use in mobile networks, it is presented in [7], that the current NFV platforms do not provide proper security and isolation to virtualized telecommunication services. One of the main challenges persistent to the use of NFV in mobile networks is the dynamic nature of VNFs that leads to configuration errors and thus security lapses [8]. Moreover, VNFs are vulnerable to typical cyber-attacks such as spoofing, sniffing and DoS. NFV is also vulnerable to a special set of virtualization threats, such as side-channel attacks, flooding attacks, hypervisor hijacking [9], malware injection, Virtual Machine (VM) migration related attacks, as well as, the cloud specific attacks. Moreover, the private deployment of NFV are vulnerable only to the malicious insiders (e.g., malicious administrator), since remote access to the system is prevented. Due to the common accessibility of the infrastructure, a malicious user or a compromised provider of VNF can interfere with the operations of the infrastructure by inserting malware or manipulating network traffic.

Operational interference and misuse of shared resources are considered as infrastructure level attacks on NFV. Due to the common accessibility of physical infrastructure resources, an attacker can interfere with operations of the infrastructure by inserting malware or manipulating network traffic. In these kind of resource misuse attacks, the victim can have no benefit of the shared or dedicated resources. The maintenance of trust in virtualized NFV systems is also a big challenge. Usually, physical network devices are installed and configured by a trusted employee and there is established trust of the device. However, VNF are fetching dynamically from the cloud, some level of trust mechanism is needed to prevent malicious VNFs. Further challenges are highlighted in Table 1.

\section{Security Challenges in Mobile Clouds and MEC}

Since cloud computing systems comprise of various resources which are shared among users, it is possible that a user spreads malicious traffic to tear down the performance of the whole system, consume more resources or stealthily access resource of other users. MEC on the other hand comprises of different complementary technologies interoperating in an open ecosystem where virtualization and distributed computing are harnessed by service providers to deploy and serve applications to end users. Given that MEC is relatively at infancy coupled with the diversity of the MEC technologies, there exists potentials for malicious attacks and privacy issues. 
Since MEC extends cloud computing capabilities to the edge of mobile networks, the level of protection that can be offered to the edge hosts is low compared to what is obtainable in traditional large data centers. Similarly, in multi-tenant cloud networks where tenants run their own control logic, interactions can cause conflicts in network configurations. Mobile Cloud Computing (MCC) migrates the concepts of cloud computing into the $5 \mathrm{G}$ eco-systems. This creates a number of security vulnerabilities that mostly arise with the architectural and infrastructural modifications in 5G. Therefore, the open architecture of MCC and the versatility of mobile terminals create vulnerabilities through which adversaries could launch threats and breach privacy in mobile clouds [10].

In this work, we categorize MCC threats according to targeted cloud segments into front-end, back-end and networkbased mobile security threats. Detailed description of each cloud segment and their threat landscapes is contained in [7]. The threat landscape at he front-end range from physical threats to application-based threats. On the back-end platform, threats are mainly targeted towards the mobile cloud servers. The scope of these threats may range from data-replication to HTTP and XML DoS (HX-DoS) attacks. For the networkbased mobile security threats, potential attacks include WiFi sniffing, DoS attacks, address impersonation, and session hijacking.

On the side of MEC, the main security concerns are in the context of cloud-enabled IoT environment as well as the open APIs through which developers and creators serve contents to MEC applications and end users. The need for open API in MEC is mainly to provide support for federated services and interactions with different providers and content creators. However the adoption of open APIs often create vulnerabilities through which adversaries in the form of third parties can launch various attacks on the MEC environment. This has triggered research on relevant security technologies channeled towards the security of the MEC nodes, which include the MEC server and other IoT nodes. Popular threats in this landscape are DoS attack, Man-in-the-Middle (MitM) attack, malicious mode problems, privacy leakages, and VM manipulation. A broad description of the threat landscape in MEC is presented in [11], here authors cover a wide array of potential security threats for the MEC system and also why security is one of the greatest challenges of MEC.

\section{Privacy Challenges in $5 G$}

From the user's perspective, the major privacy concerns could arise from data, location and identity. Most smart phone applications require details of subscriber's personal information before the installation. The application developers or companies rarely mention that how the data is stored and for what purposes it is going to be used. Threats such as semantic information attacks, timing attacks, and boundary attacks mainly target the location privacy of subscribers. At the physical layer level, location privacy can be leaked by access point selection algorithms in 5G mobile networks. International Mobile Subscriber Identity (IMSI) catching attacks can be used to reveal the identity of a subscriber by catching the
IMSI of the subscriber's User Equipment (UE). Such attacks can also be caused by setting up a fake base station which is considered as preferred base station by the UE which has lost access to Temporary Mobile Subscribers Identity (TMSI) and thus subscribers will respond with their IMSI [12].

Moreover, 5G networks have different actors such as Virtual Mobile Network Operators (VMNOs), Communication Service Providers (CSPs) and network infrastructure providers. All of these actors have different priorities for security and privacy. The synchronization of mismatching privacy policies among these actors will be a challenge in $5 \mathrm{G}$ network. In the previous generations, mobile operators had direct access and control of all the system components. However, 5G mobile operators are losing the full control of the systems as they will rely on new actors such CSPs. Thus, 5G operators will lose the full governance of security and privacy. User and data privacy are seriously challenged in shared environments where the same infrastructure is shared among various actors, for instance VMNOs and other competitors. Moreover, there are no physical boundaries of $5 \mathrm{G}$ network as they use cloud based data storage and NFV features. Hence, the $5 \mathrm{G}$ operators have no direct control of the data storing place in cloud environments. As different countries have different level of data privacy mechanisms depending upon their preferred context, the privacy is challenged if the user data is stored in a cloud in a different country.

\section{Potential Security Solutions}

In this section, we highlight security solutions for the security challenges outlined in the previous section. The challenges of flash network traffic can be solved by either adding new resources or increasing the utility of existing systems with novel technologies. We believe that new technologies such as SDN and NFV can solve these challenges more cost effectively. SDN has the capability to enable run-time resource, e.g. bandwidth, assignment to particular parts of the network as the need arises. The SDN controller can gather network stats through the south-bound API from network equipment to see if the traffic levels increase. Using NFV, services from the core network cloud can be transferred towards the edge to meet the user requirements. Similarly, NFV enables the provision virtual slices or resources at run-time to meet the growing traffic demands or surges in traffic at different network locations.

The security of the radio interface keys is still a challenge, that needs secure exchange of keys encrypted like the proposed Host Identity Protocol (HIP) based schemeS in [7]. The same end-to-end encryption protocol can be used for user plane integrity. Roaming security and network-wide mandated security policies can be achieved using centralized systems that have global visibility of the users' activities and network traffic behavior e.g. SDN. The signaling storms will be more challenging due to the excessive connectivity of UEs, small base stations, and high user mobility. C-RAN (CloudRadio Access Network) and edge computing are the potential problem solvers for these challenges, but the design of these technologies must consider the increase in signaling traffic as 
TABLE II: Potential security solutions for targeted threats [7].

\begin{tabular}{|c|c|c|c|c|c|c|}
\hline \multirow{2}{*}{ Security Solutions } & \multirow{2}{*}{ Primary Focus } & \multicolumn{3}{|c|}{ Target Technology } & \multirow{2}{*}{ Links } & \multirow{2}{*}{ Privacy } \\
\hline & & SDN & NFV & Cloud & & \\
\hline DoS, DDoS detection & Security of centralized control points & $\checkmark$ & $\checkmark$ & & & \\
\hline Configuration verification & Flow rules verification in SDN switches & $\checkmark$ & & & & \\
\hline Access control & Control access to SDN and core network elements & $\checkmark$ & $\checkmark$ & $\checkmark$ & & \\
\hline Traffic isolation & Ensures isolation for VNFs and virtual slices & & $\checkmark$ & & & \\
\hline Link security & Provide security to control channels & $\checkmark$ & & & $\checkmark$ & \\
\hline Identity verification & User identity verification for roaming and clouds services & & & & & $\checkmark$ \\
\hline Identity security & Ensure identity security of users & & & & & $\checkmark$ \\
\hline Location security & Ensure security of user location & & & & & $\checkmark$ \\
\hline IMSI security & Secure the subscriber identity through encryption & & & & & $\checkmark$ \\
\hline Mobile terminal security & Anti-maleware technologies to secure mobile terminals & & & & & $\checkmark$ \\
\hline Integrity verification & Security of data and storage systems in clouds & & & $\checkmark$ & & \\
\hline HX-DoS metigation & Security for cloud web services & & & $\checkmark$ & & \\
\hline Service access Control & Service-based access control security for clouds & & & $\checkmark$ & & \\
\hline
\end{tabular}

an important aspect of the future networks as described by NGMN. Solutions for DoS and saturation attacks, and other security challenges described in the previous section are listed in Table II and the methodologies are described below.

\section{A. Security Solutions for SDN}

Once the basic security challenges inherent in SDN are addressed, SDN can be a potential problem solver in terms of security in communication networks. Having global view of the network, centralized control and programmability in network elements, SDN enables network-wide consistent security policies and facilitates quick threat identification through a cycle of harvesting intelligence from the network resources, states and flows. Therefore, the SDN architecture supports highly reactive and proactive security monitoring, traffic analysis and response systems to facilitate network forensics, the alteration of security policies and security service insertion [13].

One of the basic feature of SDN is flow or packet level granularity that provides transparency in terms of packet origin or source, the route it takes, and even the content. Security applications can gather samples of flows or packets through the control plane from any network perimeter to check their content, regardless of the network ingress or egress ports unlike traditional networks in which the security appliances normally reside in the entry points. This capability of SDN lays the foundation for network-wide consistent security policies, early threat identification in any network location, and quick response by updating the flow tables to route traffic to Intrusion Detection Systems (IDS) or firewalls at run time. Since most of the security functionalities will be deployed in the application plane in software, security leveraging SDN can be termed as Software Defined Security [4].

\section{B. Security Solutions for NFV}

The security of VNFs through a security orchestrator in correspondence with the ETSI (European Telecommunications Standards Institute) NFV architecture is presented in [14]. The proposed architecture provides security not only to the virtual functions in a multi-tenant environment, but also to the physical entities of a telecommunication network. Using trusted computing, remote verification and integrity checking of virtual systems and hypervisors is proposed in [15] to provide hardware-based protection to private information and detect corrupt software in virtualized environments. In NFV systems, sophisticated security protection solutions such as firewalls, IDS can be used to prevent the outside attacks. Moreover, identity and access management mechanisms (e.g., role-based access control) can be used mitigate the impact of insider attacks. The infrastructure level attacks can be prevented by the continuous monitoring of the resource consumption of each users and preventing malicious requests according to a blacklist of IP addresses.

In order to increase the trust between different entities, a chain of trust relationships needs to be created and maintained in NFV environments throughout its life-cycle. Solutions based on cryptographic techniques, such as message stream encryption, can be used to guarantee the confidentiality of VNFs. Furthermore, the accountability and trust management can be utilized by VNF provider to know whether its software is running without any modification in the infrastructure providers network. Secure outsourcing is another viable solution in NFV to transfer the sensitive information to external networks. It will not only protect sensitive information but also validate the integrity of data. Moreover, security-preserving migration mechanisms establishing secure interfaces with the authorized source and destination parties, and detection and reporting of any malicious activities during the migrations are needed to enable secure VM migration.

\section{Security Solutions for Mobile Clouds and MEC}

Most proposed security measures in MCC revolve around the strategic use of virtualization technologies, the redesign of encryption methods and dynamic allocation of data processing points. Hence, virtualization comes as a natural option for securing cloud services since each end-node connects to a specific virtual instance in the cloud via a Virtual Machine (VM). Security is provided through the isolation of each user's virtual connection from other users. Virtualized in-cloud security is one of such virtulization solutions to secure MCC.

For specific security threats such as HX-DoS, specific solutions such as learning-based systems e.g. [16] are more useful than generic approaches. For example, the learningbased system take a certain number of samples of packets 
TABLE III: Security activities of various standardization bodies

\begin{tabular}{|l|l|l|l|}
\hline Standardization bodies & Workgroups & Major security areas in focus & Milestones \\
\hline $3 \mathrm{GPP}$ & $\begin{array}{l}\text { Service and System } \\
\text { Aspects Security Group } \\
\text { (SA3) }\end{array}$ & $\begin{array}{l}\text { Security architecture, RAN security, authen- } \\
\text { tication mechanism, the subscriber privacy, } \\
\text { network slicing }\end{array}$ & $\begin{array}{l}\text { TR 33.899 Study on the security aspects } \\
\text { of the next generation system, TS 33.501: } \\
\text { Security architecture and procedures for 5G } \\
\text { System }\end{array}$ \\
\hline $5 \mathrm{GPPP}$ & $5 \mathrm{GPPP}$ Security WG & $\begin{array}{l}\text { Security architecture, the subscriber privacy, } \\
\text { the authentication mechanism }\end{array}$ & $\begin{array}{l}5 \mathrm{G} \text { PPP Security Landscape (White Paper) } \\
\text { June 2017. }\end{array}$ \\
\hline IETF & $\begin{array}{l}\text { I2NSF, DICE WG, ACE } \\
\text { WG, DetNet WG }\end{array}$ & $\begin{array}{l}\text { Security solutions for massive IoT devices } \\
\text { in 5G, User privacy, Network security func- } \\
\text { tions (NSFs) }\end{array}$ & $\begin{array}{l}\text { RFC 8192, RFC 7744, Deterministic Net- } \\
\text { working (DetNet) Security Considerations }\end{array}$ \\
\hline NGMN & $\begin{array}{l}\text { NGMN 5G security group } \\
\text { (NGMN P1 WS1 5G se- } \\
\text { curity group) }\end{array}$ & $\begin{array}{l}\text { Subscriber privacy, Network slicing, MEC } \\
\text { security }\end{array}$ & $\begin{array}{l}\text { 5G security recommendations: Package 1 } \\
\text { and 2, and 5G security: Package 3 }\end{array}$ \\
\hline ETSI & $\begin{array}{l}\text { ETSI TC CYBER, ETSI } \\
\text { NFV SEC WG }\end{array}$ & $\begin{array}{l}\text { Security architecture NFV security, MEC } \\
\text { security, privacy }\end{array}$ & $\begin{array}{l}\text { ETSI GS NFV-SEC 010, ETSI GS NFV- } \\
\text { SEC 013 ETSI GS NFV-SEC 006 and ETSI } \\
\text { GS MEC 009 }\end{array}$ \\
\hline
\end{tabular}

and analyze them for various known attributes to detect and mitigate threats.

To secure the mobile terminals, anti-malware solutions are installed on the mobile terminal or hosted and served directly from the cloud. In MCC data and storage, the security framework will consist of energy efficient mechanisms for the integrity verification of data and storage services in conjunction with a public provable data possession scheme and some lightweight compromise resilient storage outsourcing. For application security, some proposed frameworks are based on securing elastic applications on mobile devices for cloud computing, lightweight dynamic credential generation mechanism for user identity protection, in-device spatial cloaking mechanism for privacy protection as well as MobiCloud which is a secure cloud framework for mobile computing and communication.

On the side of MEC, there are limited works on the side of security, however, the use of gateways at strategic points on the networks is highly recommended. IoT gateway is a typical example of such deployments. Other recommended security strategies include ensuring that the application hosted at the edge server authenticates any user attempting to access the application resources, the MEC server should be configured to protect applications and data store at the edge server from intrusion, also mobile devices should be required to authenticate the edge application accessing from the edge server and the MEC platform should give assurance of data integrity [17].

\section{Security Solutions for Privacy in $5 G$}

5G must embody privacy-by-design and service oriented privacy preserving approaches. To preserve the user privacy in $5 \mathrm{G}$ systems, there should be mutual agreements and trust models among various stakeholders involved in the process such as user, network operator, service provider, application developer, manufacturer etc on data usage and storage. Therefore, $5 \mathrm{G}$ will require better mechanisms for accountability, data minimization, transparency, openness and access control [7]. A hybrid cloud based approach is also required where mobile operators can able to store and process high sensitive data locally and less sensitive data in public clouds. In this way, operators will have more access and control over data and can decide where to share it.

For location privacy, anonymity based techniques must be applied where the subscriber real identity could be hidden and replaced with pseudonyms. Encryption based practices are useful in this case, for instance message can be encrypted before sending to Location-Based Services (LBS) provider. Techniques such as obfuscation are also crucial, where the quality of location information is reduced in order to protect location privacy. Moreover, location cloaking based algorithms are quite useful to handle some of major location privacy attacks such as timing and boundary attacks [7]. For IMSI catching attacks, one of the on-going solution for protecting the subscribers identity is to use TMSI which is generated randomly and assigned to the UE at regular intervals. The long term IMSI is utilized only in the case of fault recovery process and when TMSI is not yet allocated. Another way might be to adopt a passive method which will allow the detection of fake base stations which captures the subscriber's IMSI. Authors in [12] have proposed one of the potential solutions to protect subscribers from IMSI catching attacks in $5 \mathrm{G}$ networks.

While the standardization of $5 \mathrm{G}$, strong privacy regulations and legislation should be taken into account. The regulatory approach can be classified into three types. First is the government level regulation, where governments mainly make country-specific privacy regulations and through multi-national organizations such as the United Nations (UN) and European Union (EU). Second is the industry level, where various industries and groups such as 3GPP and ETSI collaboratively draft the best principles and practices to protect privacy. Third is the consumer level regulations where desired privacy is ensured by considering consumers' requirements [7].

\section{5G SECURITY STANDARDIZATION}

$5 \mathrm{G}$ security standardization is still in the drafting phase and various key organizations are providing immense contributions towards its rapid development, as highlighted in Table III. In March 2015, 3GPP set the deadline for defining 5G standards around 2020. The same year, NGMN published white paper [6] on 5G, that covered wide range of topics including virtualization, privacy, radio architecture, availability, and IoT among others. For 5G security standardization, the NGMN P1 
WS1 5G security group is mainly gathering requirements and provide their suggestions. In January 2016, SA3 group [3] of 3GPP started working to standardize the 5G security aspects and provide contributions to $5 \mathrm{GPPP}$ initiated projects. The major task was to propose $5 \mathrm{G}$ security architecture by analyzing threats and requirements. The SA3 group of 3GPP covers all security aspects such as RAN security, authentication mechanisms and network slicing among others [1].

The Open Networking Foundation (ONF) is dedicated to accelerate the adoption of SDN and NFV, and publishes technical specifications including specifications for security of these technologies [4]. Also, the ETSI Industry Specification Group (ISG) for NFV Security (ISG NFV SEC) is responsible for security specifications of NFV Platforms. ISG NFV SEC highlighted the need for a standard interface in ETSI NFV architecture for adding security functions that can react to potentail security threats in real-time. In 2014, ESTI MEC ISG was formed that look after MEC security standards and empowers NFV capabilities within the RAN to deliver security and robustness. NGMN 5G security group is working on identifying the security requirements for MEC and proposing corresponding recommendations. Regarding privacy, subscription privacy is one of the core security area focused in the 3GPP SA3. For example privacy enhanced identity protection deals with safeguarding the IMSI from adversaries on the air interface. SA3 is also taking valuable inputs from the FSAG group of GSMA to identify subscriber privacy challenges [1]. Furthermore, the standards suggested by the Internet Engineering Task Force (IETF) will be critical because 5G will use various Internet protocols. The International Telecommunication Union (ITU) continuously gathers contributions from regional organizations like ETSI and ARIB and proposes recommendations for the standardization organizations.

\section{CONCLUSION}

5G will use mobile clouds, SDN and NFV to meet the challenges of massive connectivity, flexibility, and costs. With all the benefits, these technologies also have inherent security challenges. Therefore, in this paper we have highlighted the main security challenges that can become more threatening in $5 \mathrm{G}$, unless properly addressed. We have also presented the potential security mechanisms and solutions for those challenges. $5 \mathrm{G}$ yet has to be deployed, thus, the security challenges in these technologies and their solutions will become more vivid. However, the integration of IoT seems to raise more security concerns, specifically in terms of privacy. Therefore, novel security solutions need to be sought out that use the development in, for example, artificial intelligence and context awareness, to enable proactive network forensics and response leveraging the programmability enabled by SDN and run-time, yet, need-based security service insertion in various network perimeters using NFV.

\section{ACKNOWLEDGMENT}

This work was supported by TEKES Finland and Academy of Finland under projects: The Naked Approach, Towards Digital Paradise and SecureConnect. Andrei Gurtov was supported by the Center for Industrial Information Technology (CENIIT).

\section{REFERENCES}

[1] 5GPPP Security WG, "5G PPP Phase1 Security Landscape, White paper," 5GPPP Papers, 2017.

[2] P. Mishra, E. S. Pilli, V. Varadharajan, and U. Tupakula, "Out-vm monitoring for malicious network packet detection in cloud," in 2017 ISEA Asia Security and Privacy (ISEASP), Jan 2017, pp. 1-10.

[3] 3GPP. (2017, May) SA3-Security. The Third Generation Partnership Project (3GPP). [Online]. Available: http://www.3gpp. org/Specifications-groups/sa-plenary/54-sa3-security

[4] I. Ahmad, S. Namal, M. Ylianttila, and A. Gurtov, "Security in Software Defined Networks: A Survey," IEEE Communications Surveys Tutorials, vol. 17, no. 4, pp. 2317-2346, Fourthquarter 2015.

[5] A. Shaik, R. Borgaonkar, N. Asokan, V. Niemi, and J. Seifert, "Practical attacks against privacy and availability in $4 \mathrm{~g} / \mathrm{lte}$ mobile communication systems," CoRR, vol. abs/1510.07563, 2015. [Online]. Available: http://arxiv.org/abs/1510.07563

[6] NGMN Alliance, "NGMN 5G white paper," Next Generation Mobile Networks, White paper, 2015.

[7] I. Ahmad, T. Kumar, M. Liyanage, J. Okwuibe, M. Ylianttila, and A. Gurtov, "5g security: Analysis of threats and solutions."

[8] W. Yang and C. Fung, "A survey on security in network functions virtualization," in 2016 IEEE NetSoft Conference and Workshops (NetSoft), June 2016, pp. 15-19.

[9] A. van Cleeff, W. Pieters, and R. J. Wieringa, "Security Implications of Virtualization: A Literature Study," in 2009 International Conference on Computational Science and Engineering, vol. 3, Aug 2009, pp. 353-358.

[10] P. Kulkarni, R. Khanai, and G. Bindagi, "Security frameworks for mobile cloud computing: A survey," in 2016 International Conference on Electrical, Electronics, and Optimization Techniques (ICEEOT), March 2016, pp. 2507-2511.

[11] R. Roman, J. Lopez, and M. Mambo, "Mobile edge computing, fog et al.: A survey and analysis of security threats and challenges," Future Generation Computer Systems, 2016.

[12] K. Norrman, M. Näslund, and E. Dubrova, "Protecting imsi and user privacy in 5g networks," in Proceedings of the 9th EAI International Conference on Mobile Multimedia Communications. ICST (Institute for Computer Sciences, Social-Informatics and Telecommunications Engineering), 2016, pp. 159-166.

[13] S. Sezer, S. Scott-Hayward, P. K. Chouhan, B. Fraser, D. Lake, J. Finnegan, N. Viljoen, M. Miller, and N. Rao, "Are we ready for SDN? Implementation challenges for software-defined networks," IEEE Communications Magazine, vol. 51, no. 7, pp. 36-43, July 2013.

[14] B. Jaeger, "Security Orchestrator: Introducing a Security Orchestrator in the Context of the ETSI NFV Reference Architecture," in 2015 IEEE Trustcom/BigDataSE/ISPA, vol. 1, Aug 2015, pp. 1255-1260.

[15] H. Lauer and N. Kuntze, "Hypervisor-based attestation of virtual environments," in 2016 Intl IEEE Conferences on Ubiquitous Intelligence Computing, Advanced and Trusted Computing, Scalable Computing and Communications, Cloud and Big Data Computing, Internet of People, and Smart World Congress (UIC/ATC/ScalCom/CBDCom/IoP/SmartWorld), July 2016, pp. 333340.

[16] A. Chonka and J. Abawajy, "Detecting and Mitigating HX-DoS Attacks against Cloud Web Services," in 2012 15th International Conference on Network-Based Information Systems, Sept 2012, pp. 429-434.

[17] A. Ahmed and E. Ahmed, "A survey on mobile edge computing," in Intelligent Systems and Control (ISCO), 2016 10th International Conference on. IEEE, 2016, pp. 1-8.

Ijaz Ahmed is a PhD student at the Centre for Wireless Communications, University of Oulu. Contact him at ijaz.ahmed@oulu.fi.

Tanesh Kumar is a PhD student at the Centre for Wireless Communications, University of Oulu. Contact him at tanesh.kumar@oulu.fi. 
Madhusanka Liyanage is a project manager at the Centre for Wireless Communications, University of Oulu. Contact him at madhusanka.liyanage@oulu.fi.

Jude Okwuibe is a PhD student at the Centre for Wireless Communications, University of Oulu. Contact him at jude.okwuibe@oulu.fi.

Mika Ylianttila is a professor at the Centre for Wireless Communications, University of Oulu. Contact him at mika.ylianttila@oulu.fi.

Andrei Gurtov is a professor at Linkping University, Sweden. Contact him at gurtov@acm.org. 